\title{
Efectos cardiovasculares debido al consumo de cannabinoides
}

\section{Oscar J. León ${ }^{\mathrm{a}, \mathrm{b}, *}$, Leonar G. Aguiar ${ }^{\mathrm{a}, \mathrm{c}, \mathrm{d}}$, Leonardo A. Quevedo ${ }^{\mathrm{a}, \mathrm{b}}$ y Ana B. Jara ${ }^{\mathrm{d}}$}

a Unidad de Urgencias, Hospital Universitario San Ignacio, Bogotá, Colombia

b Programa de Especialización en Medicina de Urgencias, Pontificia Universidad Javeriana, Bogotá, Colombia

c Departamento de Medicina Interna, Hospital Universitario San Ignacio, Bogotá, Colombia

d Facultad de Medicina, Pontificia Universidad Javeriana, Bogotá, Colombia

Recibido el 29 de marzo de 2017; aceptado el 15 de noviembre de 2017

Disponible en Internet el 20 de marzo de 2018

\author{
PALABRAS CLAVE \\ Marihuana; \\ Efectos \\ cardiovasculares; \\ Cannabis; \\ Infarto de miocardio
}

\begin{abstract}
Resumen
Objetivo: dar a conocer los efectos cardiovasculares secundarios al consumo de marihuana según lo reportado en la literatura médica.

Métodos: se realizó una búsqueda con los términos MESH "Cannabis", "Marijuana smoking" y "adverse effects" en la base de datos PubMed hasta el año 2016. Se obtuvieron 265 referencias. Se excluyeron cartas de editores, protocolos de investigación en proceso, población pediátrica (menores de 18 años), embarazadas, referencias en idiomas diferentes a inglés y español y se escogieron solo referencias relacionadas con efectos cardiovasculares.

Resultados: se han establecido dos tipos de receptores de cannabinoides, los CB1 y los CB2, con localizaciones a nivel del sistema nervioso central, endotelial, renal y músculo liso. En la actualidad el consumo de marihuana ha venido en aumento y los médicos poco conocen de sus efectos y los diferentes nombres comerciales para esta sustancia. Existen efectos protectores a nivel vascular con detención de la progresión de la placa aterosclerótica y a la vez múltiples efectos no deseados como taquicardia, hipotensión y bradicardia. Múltiples reportes de caso documentan la relación de la marihuana con el infarto agudo de miocardio con o sin lesión de las arterias coronarias, así como con hemorragia subaracnoidea, pero no existen mecanismos claramente descritos que expliquen una relación directa con estos desenlaces.

Conclusiones: se conoce la fisiopatología y los receptores donde actúan los cannabinoides generando efectos tanto protectores como dañinos. Existe fuerte correlación con enfermedad cardiovascular, principalmente síndrome coronario agudo, pero el mecanismo fisiopatológico aún no es claro.

(C) 2018 Sociedad Colombiana de Cardiología y Cirugía Cardiovascular. Publicado por Elsevier España, S.L.U. Este es un artículo Open Access bajo la licencia CC BY-NC-ND (http:// creativecommons.org/licenses/by-nc-nd/4.0/).
\end{abstract}

\footnotetext{
* Autor para correspondencia.

Correo electrónico: cayeleon1@gmail.com (0.J. León).
} 


\section{KEYWORDS}

Marihuana;

Cardiovascular

effects;

Cannabis;

Myocardial infarction

\section{Cardiovascular effects due to the use of cannabinoids}

\begin{abstract}
Objective: To determine the cardiovascular side-effects of smoking marihuana according to that reported in the medical literature.

Methods: A search was performed using the MeSH terms, "Cannabis", "Marijuana smoking" and "adverse effects", in the PubMed database up to the year 2016. A total of 265 references were obtained. The exclusion criteria were; Letters to the editor, protocols of research in process, paediatrics (less than 18 years), pregnancy, articles in languages other than English or Spanish. Only references associated with cardiovascular effects were collected.

Results: Two types of cannabinoid receptors, CB1 and CB2, have been defined and are located in the central nervous system, as well as in endothelial, renal and smooth muscle. The consumption of marijuana is increasing, and doctors know little about its effects, as well as the different marketing names used for this substance. There are protective effects at vascular level, with the slowing down of atherosclerotic plaques, as well as the many undesired effects such as, tachycardia, hypotension, and bradycardia. Many case reports document the association of marijuana with acute myocardial infarction with or without coronary artery lesions, as well as with subarachnoid haemorrhage, but there are no clearly described mechanisms that could explain a direct relationship with these events.

Conclusions: The pathophysiology is known, as well as where the cannabinoid receptors act to generate their protective and harmful effects. There is a strong association with cardiovascular disease, mainly acute coronary syndrome, but the pathophysiological is still not clear.

(c) 2018 Sociedad Colombiana de Cardiología y Cirugía Cardiovascular. Published by Elsevier España, S.L.U. This is an open access article under the CC BY-NC-ND license (http:// creativecommons.org/licenses/by-nc-nd/4.0/).
\end{abstract}

\section{Introducción}

La marihuana (Cannabis) es una de las sustancias recreativas de mayor uso en el mundo. Los cannabinoides, ingredientes activos de la marihuana, afectan múltiples órganos en el cuerpo humano. Se derivan de la planta Cannabis sativa. En tiempos atrás (en China y Medio oriente) ha sido utilizado como analgésico, relajante muscular, estimulador del apetito y broncodilatador. Contiene por lo menos 60 componentes químicos, dentro de los cuales el delta-9tetrahydrocannabinol es el producto que genera el mayor efecto biológico ${ }^{1,2}$.

Aunque los cannabinoides son unos de los componentes médicos más viejos, su química, farmacología y efectos clínicos han sido recientemente estudiados. En general, el uso de cannabinoides no se considera un riesgo para síndrome coronario agudo y se han establecido algunos beneficios en cuanto a regulación del sistema inmune y generación de aterosclerosis. Sin embargo, en la literatura científica se han documentado múltiples casos de mortalidad en cuanto a efectos cardiovasculares, así como daño neurológico y renal $^{3,4}$.

En la actualidad, el uso de cannabinoides sintéticos ha venido en aumento en respuesta a la legalización de la sustancia en varios países, lo que ha generado controversia respecto a su viabilidad y así mismo, se ha demostrado que los profesionales de la salud en el servicio de urgencias no están familiarizados con las consecuencias del consumo de marihuana, así como con los diversos nombres que se le dan a la misma ${ }^{5}$. Esta revisión de tema tiene como objetivo dar a conocer las funciones de los cannabionides en el organismo y los efectos que trae el consumo de esta sustancia con énfasis en las complicaciones cardiovasculares ${ }^{6}$.

\section{Metodología}

Se hizo una búsqueda con el término MESH "Cannabis", "Marijuana Smoking" y "adverse effects" en la base de datos PubMed hasta el año 2016. Se obtuvieron 265 referencias; se excluyeron de la selección cartas de editores, protocolos de investigación en proceso, población pediátrica (menores de 18 años), embarazadas y referencias en idiomas diferentes al inglés y al español. Se obtuvieron 48 referencias de las cuales solo se tuvieron en cuenta aquellas relacionadas con efectos cardiovasculares, para un total de 34.

\section{Epidemiología}

El cannabis es una de las sustancias ilícitas más populares en el mundo. Aproximadamente 78 millones (23.2\%) de europeos, entre los 15 y 64 años, lo han usado por lo menos una vez en la vida. En 2012, 18,9 millones (8\%) de estadounidenses usaron marihuana, reflejándose en ese año un aumento de 5 millones de usuarios en sólo 5 años $^{2,7}$.

Por otra parte, de 2006 a 2010, se evidenció que dentro de los reportes de complicaciones cardiovasculares el 1,8\% estaba asociado al uso de marihuana, presentándose una tasa de mortalidad del $25,6 \%$, de los cuales $85,7 \%$ eran hombres con promedio de edad de 34,3 años. Incluso, se ha 
considerado a la marihuana, después del tabaco y la cocaína, como una de las sustancias con mayor potencial adictivo ${ }^{3,8}$.

En 2001, Mettleman et al. aplicaron una encuesta a 3.882 pacientes cuatro días después de haber sufrido un infarto agudo de miocardio, en 64 centros médicos diferentes. De estos, 3,2\% (124 pacientes) habían fumado marihuana en el año anterior al infarto, con una edad promedio de 44 años en comparación con pacientes no fumadores quienes tenían un promedio de edad de 62 años. También se evidenció que en el grupo de fumadores de marihuana había más pacientes masculinos (94 versus 67\%), más fumadores de tabaco (68 versus $32 \%$ ) y más obesos (43 versus $32 \%$ ) en comparación con el grupo de no fumadores; sin embargo, presentaban menos episodios de angina (12 versus $25 \%)^{9}$.

Además, de los 124 pacientes que habían fumado marihuana en el último año, 67,7\% fumaban por lo menos una vez al mes y $41,2 \%$ al menos cada semana. Adicionalmente, de esos 124 pacientes, 37 fumaron dentro de las 24 horas anteriores al infarto, 9 en la hora anterior y 3 entre 60 a 120 minutos antes. Concluyen que pasada una hora de haber consumido marihuana, el riesgo de infarto agudo de miocardio aumentaba 4,8 veces (IC95\%, 2,4-9,5) ${ }^{9}$.

De otra parte, los cannabinoides sintéticos han ganado popularidad por sus efectos recreacionales, pero estos generan efectos adversos no deseados y desconocidos debido a que su contenido suele variar entre marcas y cada vez surgen más composiciones diferentes y con nuevas formas de consumo. Este último es muy frecuente en Estados Unidos; alcanza una prevalencia de 1,4 a 10\% mientras que en Europa llega a 2,8 a 4\%. Es importante tener en cuenta que puede usarse con fines terapéuticos por sus propiedades antieméticas, especialmente en el manejo de náuseas y vómito inducidos por quimioterapia. Adicionalmente, tiene propiedades analgésicas útiles en dolor neuropático ${ }^{10}$.

\section{Fisiopatología y mecanismos de acción}

Singla et al. revisaron los mecanismos de acción de los cannabinoides y sus efectos ${ }^{4}$. Existen dos receptores de membrana denominados CB1 y CB2, los cuales fueron descritos por primera vez por Mechoulam en $1995^{11}$. Estos receptores se encuentran unidos a proteína $G$ y segundos mensajeros tipo adenilato ciclasa y factor nuclear KB (NF$\mathrm{Kb})$. Los CB1 se encuentran principalmente en el cerebro y los tejidos periféricos incluyendo el endotelio, el músculo liso vascular y el riñón, en tanto que los CB2 se expresan en el sistema inmune y recientemente se han descrito en células endoteliales regulando citocinas proinflamatorias ${ }^{12}$. Dentro de las funciones de estos receptores se ha establecido que en cuanto a células del sistema inmune, se ubican en macrófagos y células T, generan una supresión del sistema inmune y disminuyen la producción de citocinas. Este efecto se ha establecido como dosis dependiente, por lo que a dosis mayores de $1 \mathrm{mg} / \mathrm{kg} /$ día el efecto es contrario y por modulación inmune celular permiten la progresión de aterosclerosis ${ }^{4}$.

A nivel endotelial, Rajesh et al. demostraron la presencia de CB1. Su estimulación generaba reactantes de fase aguda y metaloproteinasas que ayudaban a la progresión de aterosclerosis en arterias coronarias. Además, Zhao et al. descubrieron un efecto inverso con los receptores CB2 en el endotelio, información que sugiere la existencia de un efecto dicotómico ${ }^{13,14}$.

En experimentos con animales se ha demostrado disminución en la pérdida de neuronas hipocampales después de isquemia cerebral global y reducción del volumen del infarto en lesiones focales oclusivas de la arteria cerebral media en ratas. En cuanto a fisiopatología, se considera que los cannabinoides inhiben la liberación presináptica de glutamato ${ }^{15}$.

Se han visto otros efectos de forma paradójica en cuanto a la activación de los receptores y sus funciones en el metabolismo de lípidos, músculo liso vascular y zonas de reperfusión posterior a un infarto de miocardio ${ }^{4}$.

En 2012, Montecucco y Vicenzo publicaron una revisión acerca del sistema endocannabinoide y su función cardiovascular, incluidos los efectos protectores y aquellos perjudiciales para el organismo. Establecieron vías moleculares por las cuales los receptores CB1 y CB2 cumplen funciones en el endotelio y sus efectos en las arterias mesentéricas por medio de canales de calcio y segundos mensajeros, generando vasodilatación como principal mecanismo. También explica sus funciones a nivel cardiaco como efecto protector y los efectos inversos en la formación de placas de ateroma ${ }^{16}$.

\section{Efectos adversos del consumo de marihuana}

En la búsqueda de explicaciones a los efectos de la marihuana, al principio de los años setenta se realizaron estudios experimentales con animales que permitieron tener un abordaje inicial de los efectos de esta sustancia en el organismo. Dentro de los resultados obtenidos, la bradicardia es un efecto mediado por disminución de la estimulación simpática. También se estableció un efecto bifásico respecto al efecto en la tensión arterial, siendo directamente proporcional el ascenso de las cifras tensionales en relación con la dosis administrada seguido de un periodo de descenso de las mismas y de la frecuencia cardiaca ${ }^{17-19}$.

Se han descrito en la literatura diferentes efectos adversos secundarios al consumo de marihuana. En 2014, Volkow los clasificó en seis grupos, en los que se destacan alteraciones del desarrollo cerebral desde el periodo prenatal hasta los 21 años, periodo de mayor vulnerabilidad secundaria a delta-9-tetrahydrocannabinol. Establece que los efectos negativos son determinados por alteraciones en las conexiones neuronales y descenso del coeficiente intelectual. Otros efectos adversos que se describen son vulnerabilidad a otras sustancias, relación con enfermedades mentales como por ejemplo esquizofrenia, alteración en el desempeño académico, aumento del riesgo de accidentes por vehículos de motor y asociación a patologías cancerígenas ${ }^{20,21}$.

En cuanto a los efectos cardiovasculares secundarios al consumo de marihuana, Jouanjus et al. describen casos reportados en relación con su consumo en Francia, entre los años 2006 y 2010, que tuvieron relación con alteraciones cardiovasculares. En el seguimiento a los casos encontraron una tasa de mortalidad de 25,6\%; de aquellos que presentaron complicaciones cardiacas, el $90 \%$ correspondió a síndromes coronarios agudos. De 1.979 reportes analizados solo 35 $(1,8 \%)$ de los pacientes tuvo complicaciones cardiovasculares, 9 de los cuales murieron ${ }^{3}$. 
En 2000, Kunos et al. describieron los efectos cardiovasculares producidos por los endocannabinoides, principalmente la anandamida, que se une a los receptores de cannabinoides y simula muchas de las acciones biológicas de los cannabinoides derivados de las plantas. Unos de sus efectos más descritos son la hipotensión y la bradicardia, pero de menor duración de lo que se ha visto con delta-9-tetrahydrocannabinol. También describen otros endocannabinoides como el 2-araquidonolil glicerol (2-AG), pero existe poca evidencia respecto a su efecto cardiovascular y se encuentra asociado a la presencia de taquicardia, contrario a lo que puede producir la anandamida ${ }^{22,23}$.

Una revisión sistemática de Tait et al. permite establecer una relación directa en cuanto a efectos cardiovasculares y uso de cannabionoides. El efecto más frecuente es taquicardia, asociado a otros efectos no cardiovasculares como agitación y náuseas, los cuales responden bien a las medidas de soporte. Sin embargo, existen efectos severos como hemorragia subaracnoidea e infarto agudo de miocardio que requirieron intervención quirúrgica ${ }^{24}$.

En cuanto a efectos vasculares independiente de las arterias coronarias, existen múltiples series de ataque cerebrovascular asociado al consumo de marihuana en pacientes jóvenes. Ocasionalmente los síntomas fueron reversibles, lo cual parece tener mayor relación con la marihuana sintética. Aún no es claro si ese efecto está directamente relacionado con el mecanismo de acción de los cannabinoides o si ocurren por la asociación, en la mayoría de los casos, con el consumo de tabaco ${ }^{25}$.

Contrario a los efectos no deseados de la marihuana a nivel cardiovascular, también existen efectos cardioprotectores descritos. En estudios animales se produjo resistencia a la isquemia y daño por reperfusión por medio de la activación de los receptores CB2 en los miocitos. A la vez permite un efecto preventivo en la progresión de la aterosclerosis inhibiendo la migración de macrófagos dentro de los ateromas por medio de los mismos receptores. Tiene, además, un efecto antiinflamatorio en patologías cardiovasculares pero todavía no existe evidencia suficiente para atribuirlo directamente a la marihuana ${ }^{26,27}$.

En 2007, Aryana et al. establecieron los principales efectos cardiovasculares negativos asociados al consumo de marihuana (tabla 1). No obstante, hacen claridad respecto al consumo de marihuana asociado al cigarrillo, generando dificultad para establecer relación directa del consumo y los efectos causados por cada sustancia ${ }^{28}$.

En la literatura existen múltiples reportes de caso acerca de los efectos cardiovasculares y el consumo de marihuana, la mayoría en pacientes menores de 30 años, con evidencia en el cateterismo cardiaco de lesiones significativas ateromatosas que requieren intervención y, en algunos casos, revascularización miocárdica, descartando dentro de la historia clínica factores de riesgo cardiovascular ${ }^{29-32}$.

Adicional, en otros casos se ha mostrado clínica de dolor torácico o muerte súbita con estado post-reanimación los cuales se estudian para lesiones coronarias, sin evidencia de placas ateromatosas o necesidad de intervenciones quirúrgicas cardíacas, con factores de riesgo cadiovasculares evidentes o patologías vasculares genéticas. Consideran estos autores, como factor causal, posible vasoespasmo coronario dados los hallazgos imagenológicos y la evolución de los pacientes ${ }^{33-36}$.
Tabla 1 Marihuana y eventos cardiovasculares: mecanismos propuestos

Efecto proarrítmico mediado por catecolaminas. Isquemia cardiaca asociada a aumento de la frecuencia cardiaca y a trabajo cardiaco en individuos susceptibles.

Hipotensión postural.

Demora en la búsqueda de atención médica en eventos coronarios agudos por los efectos analgésicos.

Alteración en el aporte de oxígeno al corazón secundario a un aumento de los niveles de carboxihemoglobina en la sangre.

Producción de gases oxidantes, por fumar marihuana, lo cual conduce a estrés celular que puede elevar el riesgo cardiovascular por activación plaquetaria, formación de colesterol de baja densidad oxidado, cambios en la actividad del factor VII e inducción de una respuesta inflamatoria.

Marihuana y efectos cardiovasculares: Mecanismos propuestos. Adaptada de: Aryana et al., $2007^{28}$.

Casier et al. reportaron tres casos de síndrome aórtico agudo, dos de ellos con episodio de paro cardiorrespiratorio y reanimación cardiopulmonar, estudios por angiografía coronaria que mostraron aterosclerosis y requerimiento de implante de stent y revascularización. Concluyeron que a pesar de tener factores de riesgo y evidencia dentro de los estudios realizados y la enfermedad coronaria de base, el consumo de marihuana puede considerarse como un factor desencadenante de ruptura de la placa y/o vasoespasmo, asociado al aumento del consumo de oxígeno en el miocardio, así como de la tasa metabólica ${ }^{7}$.

Por último, un estudio publicado en 2013, evaluó el impacto del consumo de marihuana a largo plazo con seguimiento de los pacientes durante 18 años posterior a un infarto agudo de miocardio. No hubo una asociación estadísticamente significativa entre el consumo de marihuana previo al evento coronario y la mortalidad a los 18 años. Por lo contrario, la tasa de mortalidad fue mayor en aquellos pacientes que no consumieron marihuana, lo que justificaría los efectos protectores de los cannabinoides a nivel cardiovascular $^{37}$.

\section{Discusión}

En la actualidad el consumo de marihuana ha venido en aumento y los médicos poco conocen de sus efectos y de los diferentes nombres comerciales que existen para esta sustancia, de ahí que adquiera gran importancia conocer esta sustancia, sus derivados, el proceso fisiopatológico y las consecuencias de su consumo.

Se han establecido dos tipos de receptores de cannabinoides, los CB1 y CB2, con localizaciones en el sistema nervioso central, endotelial, renal y músculo liso. Dentro de los efectos producidos por el consumo de marihuana se han descrito procesos endoteliales e inflamatorios protectores, pero a la vez hay información respecto a efectos proinflamatorios que ayudan a la progresión de la placa. 
De igual forma, existen múltiples reportes de caso que documentan la relación de la marihuana con infarto agudo de miocardio con o sin lesión de las arterias coronarias, así como relación con hemorragia subaracnoidea; sin embargo, no existen mecanismos claramente descritos que expliquen su relación directa con estos desenlaces. La mayoría de los reportes de caso no pueden establecer como única causal de los efectos cardiovasculares la marihuana por el frecuente consumo concomitante con otras sustancias en especial el tabaco y sin descartar que en algunos casos no se evidencia lesión a nivel de las arterias coronarias.

No existe evidencia de tipo experimental, principalmente por dificultades de tipo ético, lo que dificulta dar recomendaciones acerca de su uso y contar con la experiencia suficiente para entender sus efectos. Sí existe evidencia en animales y revisiones que permiten dar una primera visión de lo que sucede desde el punto de vista fisiopatológico, pero todavía hacen falta estudios adicionales para entender mejor su utilidad y la relación riesgo/beneficio.

\section{Conclusiones}

El consumo de marihuana en la actualidad ha venido en crecimiento por la legalización de esta sustancia en varios países. Se conoce la fisiopatología y los receptores donde actúa generando efectos dicótomos, tanto protectores como dañinos, principalmente en el endotelio, al parecer, establecidos por la dosis utilizada. Existe una fuerte relación entre el consumo de marihuana y efectos cardiovasculares, específicamente, síndrome coronario agudo, si bien el mecanismo fisiopatológico no está correctamente establecido. El consumo de marihuana aumenta significativamente el riesgo de enfermedad cardiovascular, en especial si se asocia a otros factores de riesgo como consumo de tabaco y obesidad.

\section{Financiación}

Ninguna.

\section{Conflicto de intereses}

Ninguno.

\section{Bibliografía}

1. Mendizabal VE, Adler-Graschinsky E. Cannabinoids as therapeutic agents in cardiovascular disease: a tale of passions and illusions. Br J Pharmacol. 2007;151:427-40.

2. Vidot DC, Prado G, Hlaing WM, Arheart KL, Messiah SE. Emerging issues for our nation's health: the intersection of marijuana use and cardiometabolic disease risk. J Addict Dis. 2014;33:1-8.

3. Jouanjus E, Lapeyre-Mestre M, Micallef J, French Association of the Regional Abuse and Dependence Monitoring Centres (CEIPA) Working Group on Cannabis Complications*. Cannabis use: signal of increasing risk of serious cardiovascular disorders. J Am Heart Assoc. 2014;3:e000638.

4. Singla S, Sachdeva R, Mehta JL. Cannabinoids and atherosclerotic coronary heart disease. Clin Cardiol. 2012;35:329-35.

5. Lank PM, Pines E, Mycyk MB. Emergency physicians' knowledge of cannabinoid designer drugs. West J Emerg Med. 2013;14:467-70.
6. Annas GJ. Medical marijuana, physicians, and state law. N Engl J Med. 2014;371:983-5.

7. Casier I, Vanduynhoven P, Haine S, Vrints C, Jorens PG. Is recent cannabis use associated with acute coronary syndromes? An illustrative case series. Acta Cardiol. 2014;69:131-6.

8. Panayiotides IM. What is the Association of Cannabis Consumption and Cardiovascular Complications? Subst Abuse. 2015;9:1-3.

9. Mittleman MA, Lewis RA, Maclure M, Sherwood JB, Muller JE. Triggering myocardial infarction by marijuana. Circulation. 2001;103:2805-9.

10. Debruyne D, Le Boisselier R. Emerging drugs of abuse: current perspectives on synthetic cannabinoids. Subst Abuse Rehabil. 2015;6:113-29.

11. Mechoulam R, Ben-Shabat S, Hanus L, Ligumsky M, Kaminski NE, Schatz AR, et al. Identification of an endogenous 2monoglyceride, present in canine gut, that binds to cannabinoid receptors. Biochem Pharmacol. 1995;50:83-90.

12. Rajesh M, Mukhopadhyay P, Batkai S, Hasko G, Liaudet L, Huffman JW, et al. CB2-receptor stimulation attenuates TNF- -induced human endothelial cell activation, transendothelial migration of monocytes, and monocyte-endothelial adhesion. AJP: Heart and Circulatory Physiology. 2007;293: H2210-8.

13. Zhao Y, Yuan Z, Liu Y, Xue J, Tian Y, Liu W, et al. Activation of cannabinoid CB2 receptor ameliorates atherosclerosis associated with suppression of adhesion molecules. J Cardiovasc Pharmacol. 2010;55:292-8.

14. Rajesh M, Mukhopadhyay P, Hasko G, Liaudet L, Mackie K, Pacher P. Cannabinoid-1 receptor activation induces reactive oxygen species-dependent and -independent mitogen-activated protein kinase activation and cell death in human coronary artery endothelial cells. Br J Pharmacol. 2010;160:688-700.

15. Sidney S. Cardiovascular consequences of marijuana use. J Clin Pharmacol. 2002;42 11 Suppl:64S-70S.

16. Montecucco F, Di Marzo V. At the heart of the matter: the endocannabinoid system in cardiovascular function and dysfunction. Trends Pharmacol Sci. 2012;33:331-40.

17. Adams MD, Earnhardt JT, Dewey WL, Harris LS. Vasoconstrictor actions of delta8- and delta9-tetrahydrocannabinol in the rat. J Pharmacol Exp Ther. 1976;196:649-56.

18. Vollmer RR, Cavero I, Ertel RJ, Solomon TA, Buckley JP. Role of the central autonomic nervous system in the hypotension and bradycardia induced by (-)-delta 9-trans-tetrahydrocannabinol. J Pharm Pharmacol. 1974;26:186-92.

19. Brown DJ, Miller FN, Longnecker DE, Greenwald EK, Harris PD, Forney RB. The influence of delta 9-tetrahydrocannabinol on cardiovascular and subcutaneous microcirculatory systems in the bat. J Pharmacol Exp Ther. 1974;188:624-9.

20. Volkow ND, Baler RD, Compton WM, Weiss SR. Adverse health effects of marijuana use. N Engl J Med. 2014;370: 2219-27.

21. Thomas G, Kloner RA, Rezkalla S. Adverse cardiovascular, cerebrovascular, and peripheral vascular effects of marijuana inhalation: what cardiologists need to know. Am J Cardiol. 2014;113:187-90.

22. Kunos G, Jarai Z, Varga K, Liu J, Wang L, Wagner JA. Cardiovascular effects of endocannabinoids-the plot thickens. Prostaglandins Other Lipid Mediat. 2000;61(1-2):71-84.

23. Randall MD, Kendall DA, O'Sullivan S. The complexities of the cardiovascular actions of cannabinoids. $\mathrm{Br} \mathrm{J}$ Pharmacol. 2004;142:20-6.

24. Tait RJ, Caldicott D, Mountain D, Hill SL, Lenton S. A systematic review of adverse events arising from the use of synthetic cannabinoids and their associated treatment. Clin Toxicol (Phila). 2016;54:1-13.

25. Rezkalla S, Stankowski R, Kloner RA. Cardiovascular effects of marijuana. J Cardiovasc Pharmacol Ther. 2016;21:452-5. 
26. Steffens S, Veillard NR, Arnaud C, Pelli G, Burger F, Staub C, et al. Low dose oral cannabinoid therapy reduces progression of atherosclerosis in mice. Nature. 2005;434:782-6.

27. Franz CA, Frishman WH. Marijuana use and cardiovascular disease. Cardiol Rev. 2016;24:158-62.

28. Aryana A, Williams MA. Marijuana as a trigger of cardiovascular events: Speculation or scientific certainty? Int J Cardiol. 2007;118:141-4.

29. Tatli E, Yilmaztepe M, Altun G, Altun A. Cannabis-induced coronary artery thrombosis and acute anterior myocardial infarction in a young man. Int J Cardiol. 2007;120:420-2.

30. Velibey Y, Sahin S, Tanik O, Keskin M, Bolca O, Eren M. Acute myocardial infarction due to marijuana smoking in a young man: guilty should not be underestimated. Am J Emerg Med. 2015;33, 1114e1-1114.e3.

31. Yurtdas M, Aydin MK. Acute myocardial infarction in a young man; fatal blow of the marijuana: a case report. Korean Circ J. 2012;42:641-5.
32. Lindsay AC, Foale RA, Warren O, Henry JA. Cannabis as a precipitant of cardiovascular emergencies. Int J Cardiol. 2005; 104:230-2.

33. Sattout AH, Nicol MF. Cardiac arrest following cannabis use: a case report. Cases J. 2009;2:208-1626.

34. Safaa AM, Markham R, Jayasinghe R. Marijuana-induced recurrent acute coronary syndrome with normal coronary angiograms. Drug Alcohol Rev. 2012;31:91-4.

35. Menahem S. Cardiac asystole following Cannabis (marijuana) usage-additional mechanism for sudden death? Forensic Sci Int. 2013;233(1-3):e3-5.

36. Hodcroft CJ, Rossiter MC, Buch AN. Cannabis-associated myocardial infarction in a young man with normal coronary arteries. J Emerg Med. 2014;47:277-81.

37. Frost L, Mostofsky E, Rosenbloom JI, Mukamal KJ, Mittleman MA. Marijuana use and long-term mortality among survivors of acute myocardial infarction. Am Heart J. 2013;165: $170-5$. 